\title{
Primeiro registro documentado de Holochilus brasiliensis (Desmarest, 1819) e Calomys laucha (G. Fischer, 1814) no estado de Santa Catarina, sul do Brasil
}

\author{
Felipe Bortolotto Peters * \\ Paulo Ricardo de Oliveira Roth \\ Alexandre Uarth Christoff \\ Departamento de Biologia, Museu de Ciências Naturais, Universidade Luterana do Brasil \\ Avenida Farroupilha, 8001, CEP 92425-900, Canoas - RS, Brasil. \\ * Autor para correspondência \\ felipe.peters@areadevida.com.br
}

Submetido em 24/10/2012

Aceito para publicação em 05/07/2013

\section{Resumo}

Estudos sobre riqueza de pequenos mamíferos na região sul do Brasil são recentes e apontam a necessidade do uso de métodos variados. Nesse sentido, este artigo contribui para o conhecimento da fauna de pequenos roedores sigmodontíneos de Santa Catarina, incluindo Holochilus brasiliensis e Calomys laucha na listagem de espécies com ocorrência confirmada nesse estado. Os registros de $H$. brasiliensis referem-se a Laguna $(\mathrm{n}=2)$ e Timbé do Sul $(\mathrm{n}=1)$. Já $C$. laucha apresenta 23 registros provenientes de Capinzal. Todo o material é representado por fragmentos cranianos provenientes de amostras de egagrópilos coletadas em ninhos e poleiros de alimentação da coruja-de-igreja (Tyto alba).

Palavras-chave: Bioma Mata Atlântica; Egagrópilos; Sigmodontíneos; Tyto alba

\section{Abstract}

First documented record of Holochilus brasiliensis (Desmarest, 1819) and Calomys laucha (G. Fischer, 1814) in the state of Santa Catarina, south Brazil. Studies on the richness of small mammals in the Brazilian South Region are recent and point out the need for using varied methods. This way, this paper contributes to knowledge on the fauna of small sigmodontinae rodents in Santa Catarina, including Holochilus brasiliensis and Calomys laucha in the list of species with confirmed occurrence in this state. The records of H. brasiliensis refer to Laguna $(\mathrm{n}=2)$ and Timbe do Sul $(\mathrm{n}=1)$. In turn, C. laucha presents 23 records from Capinzal. The entire material is represented by skull fragments from owl pellets samples collected from nests and feeding perches of the Tyto alba.

Key words: Atlantic Forest biome; Owl pellets; Sigmodontinae; Tyto alba 


\section{Introdução}

Os roedores muroideos do Novo Mundo pertencentes à subfamília Sigmodontinae representam um dos grupos mais diversos e complexos de mamíferos. Totalizam aproximadamente 377 espécies formalmente descritas no âmbito mundial (sensu MUSSER; CARLETON, 2005), das quais cerca de 131 já foram registradas em território brasileiro (PAGLIA et al., 2012). Todos estes números representam uma subestimativa dos valores atuais, visto que o conhecimento sobre a riqueza de pequenos roedores na América do Sul, incluindo o sul do Brasil, ainda é deficiente (PATTERSON, 2000).

Em Santa Catarina (SC), o desenvolvimento de trabalhos abordando a diversidade da mastofauna foi crescente ao longo dos últimos anos. Destacam-se os estudos elaborados por Azevedo et al. (1982), Cimardi (1996), Avila-Pires (1999), Graipel et al. (2001), Cherem et al. $(2004 ; 2011)$ e Cherem (2005). Assumindo o trabalho de Cherem et al. (2004) como a referência mais completa e atual dos mamíferos do Estado de $\mathrm{SC}$, verifica-se o registro de 20 espécies de roedores sigmodontíneos com ocorrência confirmada através de material testemunho e 15 espécies com ocorrência potencial.

Recentemente novos táxons têm sido estabelecidos, assim como ampliações de áreas de distribuição continuam a ser registradas. Nessa situação estão Akodon serrensis, Abrawayaomys ruschii, Drymoreomys albimaculatus, Rhagomys rufescens e Rhipidomys mastacalis, atualmente confirmados para o território catarinense (CHEREM et al., 2011; PASSAMANI et al., 2011; PERCEQUILLO et al., 2011; TESTONI et al., 2012).

Holochilus brasiliensis e Calomys laucha apresentam potencialidade da ocorrência para SC, no entanto, atualmente não há confirmação do estabelecimento dessas duas espécies no Estado (CHEREM et al., 2004). Holochilus brasiliensis, assumindo $H$. vulpinus como sinônimo (sensu MUSSER; CARLETON, 2005), é um sigmodontíneo de grande porte, herbívoro, noturno e de hábito locomotor semiaquático (ACHAVAL et al., 2007). Apresenta registro para o centro-leste da Argentina, Uruguai e sul-sudeste do Brasil (MUSSER; CARLETON, 2005), onde seus limites permanecem obscuros, devido à falta de amostras adequadas e à carência de estudos recentes (VIVO et al., 2010). Já C. laucha é um sigmodontíneo de pequeno porte, noturno, predominantemente herbívoro (BARLOW, 1969) e de hábito locomotor cursorial (BONVICINO et al., 2008). Apresenta registro no centro-sul da Bolívia, norte e centro-leste da Argentina, oeste do Paraguai, centro-oeste e extremo sul do Brasil e Uruguai (MUSSER; CARLETON, 2005; BONVICINO et al., 2008). Considerando essas informações, este trabalho contribui para o conhecimento da fauna de pequenos mamíferos de $\mathrm{SC}$, incluindo $H$. brasiliensis e C. laucha na listagem de espécies com ocorrência confirmada para este Estado.

\section{Material e Métodos}

Os registros de H. brasiliensis e C. laucha são provenientes de amostras pontuais de egagrópilos coletados em ninhos e poleiros de alimentação da coruja-de-igreja (Tyto alba) (Aves, Tytonidae) em três municípios catarinense. A primeira coleta ocorreu na zona urbana de Timbé do Sul (2849'55”S/4950’48”O) em 23 de junho de 2010. A segunda ocorreu na localidade de Magalhães, zona urbana de Laguna (2829'35"S/48 46'36"O) em 24 de setembro de 2010. Já a terceira é proveniente da área periurbana do município de Capinzal (27²0’59’S/51³7’60”O) e ocorreu em 14 de dezembro de 2011.

Todos os pontos de coleta estão inseridos em áreas alteradas em decorrência da expansão agropecuária ou da malha urbana. Os elementos vegetacionais observados no entorno de Timbé do Sul e Laguna correspondem à formação de Floresta Ombrófila Densa, enquanto Capinzal apresenta elementos típicos de Floresta Ombrófila Mista (sensu IBGE, 2004) (Figura 1).

Os ambientes locais potenciais para a ocorrência de C. laucha e H. brasiliensis foram verificados a partir da caracterização da fitofisionomia do entorno dos sítios amostrais. Os limites foram definidos com base no perímetro de caça do predador. Neste caso, foi determinado um raio de até cinco quilômetros a partir do poleiro (sensu TAYLOR, 1994), visto que a coruja- 
de-igreja pode apresentar comportamento oportunista, deslocando-se para caçar em áreas com melhor oferta de presas (VELARDE et al., 2007).

FIGURA 1: Localização dos municípios com registros inéditos de Holochilus brasiliensis (A e B) e Calomys laucha (C) obtidos em egagrópilos de Tyto alba (coruja-deigreja) no Estado de Santa Catarina, sul do Brasil. A - Timbé do Sul; B - Laguna; C - Capinzal.

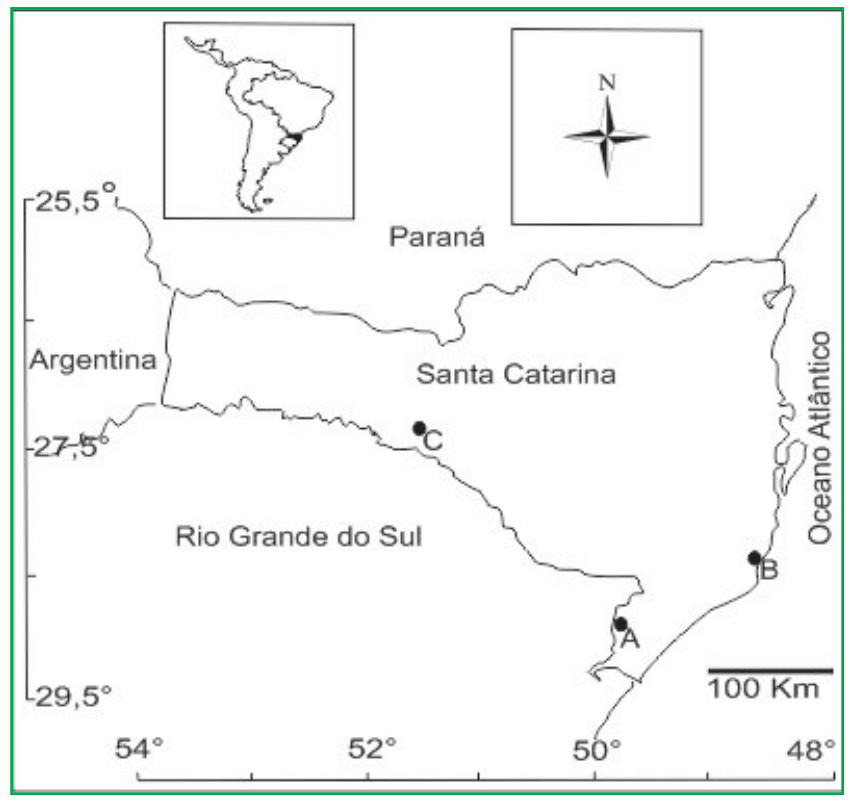

Todo o material foi triado em laboratório e individualizado a partir da contagem do número mínimo de fragmentos hemimandibulares encontrados nas pelotas. As características craniodentárias utilizadas para determinação específica de $H$. brasiliensis e $C$. laucha seguem os trabalhos de Hershkovitz (1955; 1962), Christoff et al. (no prelo) e Weksler et al. (2006, exclusivamente para $H$. brasiliensis). As demais espécies registradas nas amostras foram identificadas através de comparação com o acervo de referência da Coleção de Mamíferos do Museu de Ciências Naturais da Universidade Luterana do Brasil. Todo o material testemunho encontra-se tombado na Coleção de Fragmentos (MCNU-CF) da referida instituição. As citações taxonômicas seguem os arranjos de Musser e Carleton (2005), sendo a única exceção aplicada ao gênero Oryzomys. Neste caso, é aceita a revisão atual de Weksler et al. (2006).

As medidas craniodentárias resgatadas foram aferidas sob estereomicroscópio, utilizando um paquímetro digital com precisão de $0,01 \mathrm{~mm}$ (sensu VOSS, 1991). São apresentados comprimento da fossa orbital (CFO), comprimento do diastema (CD), comprimento do forâmen incisivo (CFI), comprimento da série molar superior (CSM), largura mínima da região interorbital (LMI), largura da caixa craniana (LC), largura do rostro (LR), largura da ponte palatal (LPP), largura da placa zigomática (LPZ), largura do primeiro molar superior (LM1) e diâmetro incisivo (DI).

\section{Resultados}

Os registros de $H$. brasiliensis são apresentados para Timbé do Sul $(n=1)$ e Laguna $(n=2)$. A determinação específica ocorreu a partir da constatação das seguintes características craniodentárias: rostro curto, nasais curvados à região ventral; placa zigomática larga apresentando processo espinhoso; região interorbital muito estreita com cristas conspícuas; arcos zigomáticos robustos e largos; fossa mesopterigoidea estreita e bicôncava; molares hipsodontes, planos e providos de cúspides ovais/subtriangulares; primeiro molar superior com mesolofo vestigial e muro mediano conectando o paracone; terceiro molar inferior sem protoflexídeo (HERSHKOVITZ, 1955; WEKSLER et al., 2006; CHRISTOFF et al., no prelo) (Figura 2). Todos os exemplares apresentam série molar com desgaste muito reduzido, indicando tratarem-se de indivíduos jovens. As medidas craniodentárias tomadas de $H$. brasiliensis correspondem às variáveis verificadas por Hershkovitz (1955) e Marques (1988) para exemplares coletados no Uruguai e Rio Grande do Sul, respectivamente (Tabela 1).

Apenas um exemplar de $H$. brasiliensis foi identificado na amostra de Timbé do Sul, junto com Oligoryzomys flavescens $(\mathrm{n}=45)$, Oligoryzomys nigripes $(\mathrm{n}=12)$, Thaptomys nigrita $(\mathrm{n}=2)$, Mus musculus $(\mathrm{n}=14)$ e Rattus rattus $(\mathrm{n}=11)$. O sítio amostral limita-se ao norte e oeste pela encosta da Serra Geral, local de maior altitude (300 m) e concentração florestal, que apresentam condições geográficas não relacionadas à presença de H. brasiliensis (sensu BARLOW, 1969; ACHAVAL et al., 2007; GONZÁLES; LANFRANCO, 2010). Ao sul e leste ocorrem vastas planícies de inundação de influência fluvial (100 $\mathrm{m}$ de altitude), formando restingas 
FIGURA 2: Fragmentos cranianos de Calomys laucha (A) e Holochilus brasiliensis (B) resgatados de egagrópilos de Tyto alba no Estado de Santa Catarina, sul do Brasil. A- Exemplar MCNU-CF-1897 em vista dorsal e MCNU-CF-1890 em vista ventral, ambos coletados em Capinzal. B- Exemplar MCNU-CF-801 em vista dorsal e MCNU-CF-387 vista ventral, coletados em Laguna e Timbé do Sul, respectivamente.

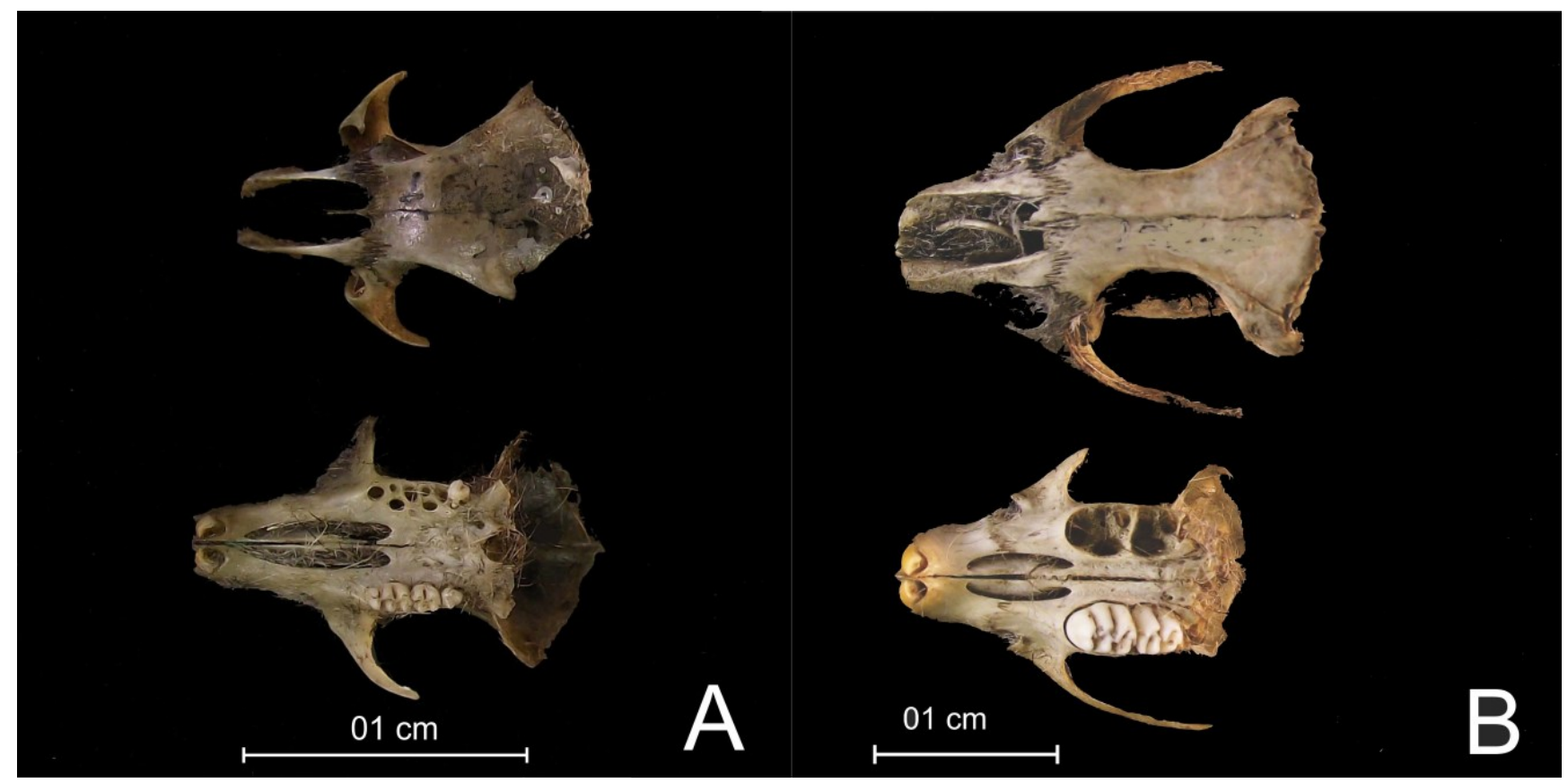

e banhados, onde o regime hídrico é determinado pela prática da orizicultura. Apesar do impacto agrícola, estes ambientes são tipicamente relacionados à presença de $H$. brasiliensis, o qual pode obter vantagem frente à disponibilidade de grãos cultivados (MARQUES, 1988). Segundo Hershkovitz (1955), a espécie ocupa áreas alagadas com abundante vegetação herbáceoarbustiva. Nesses locais escava galerias nas margens (MASSOIA, 1971) ou constroi ninhos com folhas, ramos e outros restos vegetais pouco acima da linha d'água (SAUTHIER et al., 2010).

Outros dois indivíduos de $H$. brasiliensis foram identificados na amostra de Laguna, junto com $O$. nigripes $(\mathrm{n}=2), M$. musculus $(\mathrm{n}=1)$ e $R$. rattus $(\mathrm{n}=3) . \mathrm{O}$ sítio amostral limita-se ao norte pelo Morro da Glória, local de maior altitude (100 m) e concentração florestal que apresenta condições geográficas incompatíveis para a presença da espécie (sensu BARLOW, 1969; ACHAVAL et al., 2007; GONZÁLES; LANFRANCO, 2010). Ao sul e a oeste localiza-se o Canal da Barra e a Lagoa de Santo Antônio, respectivamente. Estas áreas úmidas apresentam influência marinha, onde é possível verificar manguezais e marismas. Os ambientes relacionados à presença de $H$. brasiliensis estão localizados ao leste do perímetro, onde ocorrem as planícies de inundação ( 8 $\mathrm{m}$ de altitude) de influência fluvial, formando restingas e brejos de água doce (FERREIRA, 2006).

O registro inédito de espécimes relacionados a C. laucha ocorreu na amostra de Capinzal. A determinação específica ocorreu a partir da constatação das seguintes características craniodentárias: presença de bordas supraorbitais divergentes, margens quadradas, nunca frisadas; interparietal usualmente estreito anteroposteriormente; molares simples, mesolofo ausente, com flexo anteromediano presente; molares superiores com cônulo anterolingual menor do que o anterolabial (HERSHKOVITZ, 1962; CHRISTOFF et al., no prelo) (Figura 2). A classe etária dos indivíduos, aferida através do desgaste da série molar, aponta para o registro de adultos em cerca de $70 \%$ da amostra analisada. As medidas aferidas para C. laucha correspondem aos valores verificados por Hershkovitz (1962) para exemplares coletados no Uruguai (Tabela 1).

Vinte e três exemplares de C. laucha foram identificados junto com Gracilinanus microtarsus 
TABELA 1: Medidas craniodentárias $(\mathrm{mm})$ resgatadas dos exemplares de Calomys laucha e Holochilus brasiliensis coletados em egagrópilos de Tyto Alba, no Estado de Santa Catarina, sul do Brasil.

\begin{tabular}{|c|c|c|c|c|c|c|c|c|c|c|c|}
\hline Espécie/Tombo & CFO & LMI & $\mathbf{L C}$ & CD & LR & LPP & CFI & LPZ & DI & CSM & LM1 \\
\hline \multicolumn{12}{|l|}{ C. laucha* } \\
\hline MCNU-CF-1891 & - & 3,17 & - & 5,33 & 3,41 & 2,58 & 5,29 & 1,95 & 1,08 & 3,34 & 0,91 \\
\hline MCNU-CF-1892 & - & 3,63 & - & 5,09 & 3,05 & 2,48 & 4,70 & 2,11 & 1,23 & 3,18 & 0,89 \\
\hline MCNU-CF-1893 & - & 3,53 & - & 5,41 & 3,54 & 2,76 & 5,09 & 2,21 & 1,08 & 3,27 & 1,08 \\
\hline MCNU-CF-1894 & - & 3,47 & - & 5,21 & 3,52 & 2,60 & 4,54 & 1,95 & 1,16 & 3,06 & 0,90 \\
\hline MCNU-CF-1895 & - & 3,58 & - & 4,70 & 3,71 & 2,72 & 4,74 & 1,92 & - & - & 0,96 \\
\hline MCNU-CF-1896 & - & 3,22 & - & 5,04 & 3,45 & 2,84 & 4,62 & 2,15 & - & 3,22 & 0,92 \\
\hline MCNU-CF-1897 & - & 3,34 & - & 4,85 & 3,38 & 2,71 & 4,48 & 1,90 & - & - & 0,90 \\
\hline MCNU-CF-1898 & - & 3,47 & - & 5,22 & - & - & 4,84 & 2,06 & - & - & 0,84 \\
\hline MCNU-CF-1899 & - & 3,17 & - & 5,00 & 3,16 & 2,37 & 4,47 & 1,83 & - & 3,05 & 0,84 \\
\hline MCNU-CF-1900 & - & 3,28 & - & 5,58 & 3,42 & 2,42 & 5,02 & 2,28 & - & 3,15 & 0,89 \\
\hline MCNU-CF-1901 & - & 3,25 & - & 5,14 & 3,42 & 2,35 & 5,03 & 2,08 & - & 3,02 & 0,85 \\
\hline MCNU-CF-1902 & - & 3,45 & - & 4,86 & 3,35 & 2,22 & 4,45 & 2,09 & - & 3,17 & 0,95 \\
\hline MCNU-CF-1903 & - & 3,32 & - & 4,82 & - & - & 5,06 & 2,15 & 1,1 & - & - \\
\hline MCNU-CF-1904 & - & 3,50 & - & 4,90 & 3,48 & 2,34 & 4,67 & 2,13 & 1,17 & 3,39 & 0,96 \\
\hline MCNU-CF-1905 & - & 3,47 & - & 5,55 & 3,48 & 2,44 & 5,29 & 2,19 & & 3,2 & 0,93 \\
\hline MCNU-CF-1906 & - & 3,52 & - & 5,18 & 3,24 & 2,44 & 4,71 & 2,00 & 1,24 & - & 0,86 \\
\hline MCNU-CF-1907 & - & - & - & - & - & - & - & 2,09 & - & 3,24 & 0,88 \\
\hline MCNU-CF-1908 & - & - & - & - & - & - & - & & - & 3,26 & 0,93 \\
\hline MCNU-CF-1909 & - & - & - & - & - & - & - & 2,09 & - & - & 0,95 \\
\hline MCNU-CF-1910 & - & - & - & 5,29 & - & - & 4,96 & 2,18 & - & - & 0,90 \\
\hline MCNU-CF-1911 & - & - & - & - & - & - & - & 2,1 & - & 3,36 & 0,94 \\
\hline MCNU-CF-1912 & - & - & - & - & - & - & - & 2,15 & - & 3,03 & 0,88 \\
\hline MCNU-CF-1913 & - & - & - & - & - & - & - & 2,08 & - & 3,20 & 0,93 \\
\hline \multicolumn{12}{|l|}{ H. brasiliensis } \\
\hline MCNU-CF-387** & - & 4,03 & - & 6,81 & 5,48 & 1,89 & 5,22 & 2,58 & - & - & 2,27 \\
\hline MCNU-CF-801*** & - & 4,38 & - & 9,04 & 5,83 & 2,27 & 6,61 & 3,71 & 1,67 & 7,62 & 2,40 \\
\hline MCNU-CF-802*** & 13,87 & 4,12 & 14,31 & 10,15 & 6,20 & 2,09 & 7,31 & 4,37 & 1,70 & - & 2,22 \\
\hline
\end{tabular}

*Coletados em Capinzal; **Coletado em Timbé do Sul; ***Coletados em Laguna.

$(\mathrm{n}=1)$, Monodelphis sp. $(\mathrm{n}=4)$, Akodon paranaensis ( $\mathrm{n}=48)$, Akodon $\mathrm{cf}$. montensis ( $\mathrm{n}=11)$, Brucepattersonius iheringi $(\mathrm{n}=6)$, Necromys lasiurus $(\mathrm{n}=9)$, O. flavescens $(\mathrm{n}=37)$, O. nigripes $(\mathrm{n}=245)$, Oxymycterus quaestor $(\mathrm{n}=3)$, Sooretamys angouya $(\mathrm{n}=6)$, T. nigrita $(\mathrm{n}=3), M$. musculus $(\mathrm{n}=182)$ e $R$. rattus $(\mathrm{n}=8)$. A área do entorno do sítio de coleta caracteriza-se pela presença de coxilhas intercaladas por depressões variando entre $450 \mathrm{~m}$ de altitude no leito do rio do Peixe e $700 \mathrm{~m}$ de altitude no topo da encosta. As sedes municipais de Capinzal e Ouro estão localizadas a nordeste, áreas urbanizadas e em condições ambientais incompatíveis para a presença de $C$. laucha. Nos demais quadrantes encontram-se fragmentos florestais relictuais inseridos em uma matriz campestre alterada, em decorrência da agropecuária e de pequenos estabelecimentos humanos. Segundo Hershkovitz (1962), C. laucha ocupa estas formações campestres secas, utilizando também touceiras de capim e encostas rochosas como abrigo (GONZÁLES; LANFRANCO, 2010). Kravetz et al. (1981) ainda relatam a tolerância na ocupação de ambientes peridomicilares ou modificados pela agricultura e pecuária.

\section{Discussão}

Hershkovitz (1955) apresentou uma ampla distribuição geográfica de $H$. brasiliensis para o território 
brasileiro, excluindo apenas o extremo noroeste da Amazônia. No entanto, estudos posteriores revelaram que o táxon referido por Hershkovitz (1955) abrange um complexo de três ou mais espécies (GARDNER; PATTON, 1976; MASSOIA, 1980; 1981; AGUILERA; PÉREZ-ZAPATA, 1989), sendo a distribuição geográfica de H. brasiliensis (sensu stricto) posteriormente restringida por Massoia (1980; 1981).

Holochilus brasiliensis tem Lagoa Santa, Minas Gerais como localidade-tipo (HERSHKOVITZ, 1955). Demais registros são confirmados para Espírito Santo (BONVICINO et al., 2008; OLIVEIRA; BONVICINO, 2011), São Paulo (VIVO et al., 2010); Paraná (BONVICINO et al., 2009) e Rio Grande do Sul (SILVA, 1994; PETERS et al., 2010). Bonvicino et al. (2008) consideraram como potencial a ocorrência da espécie para a região central catarinense, excluindo apenas a região litorânea e o extremo oeste do Estado. Cherem et al. (2004) consideraram H. brasiliensis como espécie de possível ocorrência para SC. Posteriormente, Cherem et al. (2011) relatam a captura de quatro espécimes atribuídos ao gênero Holochilus, mas sem a determinação do táxon específico.

Hershkovitz (1962) revisou o gênero Calomys, reconhecendo $C$. laucha e outras três espécies. Estudos subsequentes verificaram que C. laucha, bem como C. callosus, tratavam-se de um complexo de espécies (MASSOIA et al., 1968; PEARSON; PATTON, 1976; WILLIAMS; MARES, 1978; CORTI et al., 1987; BONVICINO; ALMEIDA, 2000; BONVICINO et al., 2010). Atualmente, 13 táxons específicos são reconhecidos, sendo sete já registrados para o Brasil (MUSSER; CARLETON, 2005; BONVICINO et al., 2010). A ocorrência de C. laucha em território brasileiro é citada para as regiões sul e centro-oeste (MUSSER; CARLETON, 2005). No entanto, esta informação não é reconhecida em Bonvicino et al. (2008; 2010) e Paglia et al. (2012), que restringem a espécie ao bioma Pampa. Recentemente Peters et al. (2010) e Badzinski et al. (2012) ampliaram a distribuição de C. laucha, ao apresentarem ocorrências que atingem o bioma Mata Atlântica no Estado do Rio Grande do Sul.

Os registros de C. laucha e H. brasiliensis representam a primeira ocorrência documentada destas espécies de hábitos campestres no Estado de SC. Formoso et al. (2010) citaram a possibilidade de relação entre o aumento das áreas irrigadas por canais artificiais e a disponibilidade de grãos, sobretudo do arroz, com a dispersão de $H$. brasiliensis para novos locais. Já Kravetz et al. (1981) ressaltaram a tolerância de C. laucha na ocupação de áreas peridomiciliares e de ambientes alterados pela agricultura e pecuária. Ambas as considerações poderiam estar favorecendo $C$. laucha $\mathrm{e}$ H. brasiliensis na colonização de novas áreas campestres. Aparentemente esta situação poderia ser decorrente da atual diminuição do estrato florestal originalmente dominante no bioma Mata Atlântica de SC.

A ausência de espécies de interesse conservacionista e a presença de murídeos compondo a comunidade de roedores amostrados refletem a atual degradação das condições ambientais de todos os sítios analisados, os quais estão inseridos próximos ou mesmo no interior de centros urbanos. Este fato ressalta a plasticidade ecológica de $C$. laucha e H. brasiliensis, os quais podem estar obtendo vantagens em relação à disponibilidade de alimento de origem humana, à fragmentação dos habitats florestais e à consequente redução de ameaça por predadores terrestres.

A questão da escassez de inventários direcionados à mastofauna também deve ser ponderada. A grande maioria dos estudos direcionados a pequenos roedores parte da utilização de armadilhas não-letais convencionais (e.g. Sherman, Tomahawk, gaiolas de arame). A utilização de apenas um método pode não ser suficiente para o sucesso amostral, já que espécies herbívoras, sinantrópicas ou com menor densidade populacional podem não ser detectadas com armadilhas que utilizam iscas. $\mathrm{O}$ maior exemplo trata justamente de C. laucha e H. brasiliensis, nas quais o hábito alimentar herbívoro pode ser um empecilho à captura, resultando na subamostragem verificada.

O conhecimento pleno sobre a riqueza de pequenos mamíferos na região sul do Brasil passa pela necessidade de levantamentos em longo prazo e com o uso de métodos variados (CHEREM et al., 2011). O fato de os sítios de coleta dos egagrópilos de T. alba estarem localizados em áreas urbanas ou peridomiciliares demonstra que o método é eficiente e independe das 
condições ambientais encontradas imediatamente no entorno do abrigo. Os registros inéditos de C. laucha e $H$. brasiliensis corroboram com as discussões e resultados de Bonvicino e Bezerra (2003), Scheibler e Christoff (2007), Peters et al. (2010) e Rocha et al. (2011), os quais ressaltam a importância do método na investigação da riqueza local de pequenos mamíferos, compensando o esforço na localização dos abrigos do predador e posterior triagem laboratorial.

\section{Agradecimentos}

A Edson Fiedler de Abreu-Junior, Osvaldo Onghero Júnior e Eduardo de Lima Coelho pela ajuda em campo e laboratório. Aos revisores pelas valiosas contribuições ao manuscrito. Ao Conselho Nacional de Desenvolvimento Científico e Tecnológico (CNPq) (Processos 464797/2000-4 e 471551/2010-4) pela concessão de Bolsas de Iniciação Científica e à PróReitoria de Pesquisa e Pós-Graduação da Universidade Luterana do Brasil pelo apoio institucional.

\section{Referências}

ACHAVAL, F.; ClARA, M.; OLMOS, A. Mamíferos de la República Oriental del Uruguay. 2. ed. Montevideo: Imprimex, 2007. 216 p.

AGUILERA, A.; PÉREZ-ZAPATA, A. Cariologia de Holochilus venezuelae (Rodentia, Cricetidae). Acta Científica Venezolana, Caracas, v. 40, n. 1, p. 198-207, 1989.

AVILA-PIRES, F. D. Mamíferos descritos do Estado de Santa Catarina. Revista Brasileira de Zoologia, Curitiba, v. 16, n. 2, p. 51-62, 1999.

AZEVEDO, T. R.; ACHKAR, D. E. L.; MARTINS, M. F.; XIMENEZ, A. Lista sistemática dos mamíferos de Santa Catarina conservados nos principais museus do estado. Revista Nordestina de Biologia, João Pessoa, v. 5, n. 1, p. 93-104, 1982.

BADZINSKI, C.; GALIANO, D.; MARINHO, J. R. Mammalia, Rodentia, Cricetidae, Calomys laucha (Fischer, 1814): distribution extension in southern Brazil. Check List, Viçosa, v. 8, n. 2, p. 264266, 2012.

BARLOW, J. C. Observations on the biology of rodents in Uruguay. Life Sciences Contributions, Ontario, v. 75, n. 1, p. 1-59, 1969.

BONVICINO, C. R.; ALMEIDA, F. C. Karyotype, morphology and taxonomic status of Calomys expulsus (Rodentia: Sigmodontinae). Mammalia, Munchen, v. 64, n. 3, p. 339-351, 2000.

BONVICINO, C. R.; BEZERRA, A. M. Use of regurgited pellets of barn owl (Tyto alba) for inventorying small mammals in the Cerrado of central Brazil. Studies on Neotropical Fauna and Environment, Tübbingen, v. 38, n. 1, p. 1-5, 2003.
BONVICINO, C. R.; OLIVEIRA, J. A.; D'ANDREA, P. S. Guia dos roedores do Brasil, com chaves para gêneros baseadas em caracteres externos. Rio de Janeiro: Centro Pan-Americano de Febre Aftosa - OAS/OMS, 2008. 120 p.

BONVICINO, C. R.; OLIVEIRA, J. A.; GENTILE, R. A new species of Calomys (Rodentia: Sigmodontinae) from eastern Brazil. Zootaxa, Auckland, v. 2336, n. 1, p. 19-25, 2010.

BONVICINO, C. R; REIS, N. R.; ROSANEIS, B. K.; FREGONEZI, M. N. Ordem Rodentia. In: REIS, N. R.; PERACCHI, A. L.; FREGONEZI, M. N.; ROSANEIS, B. K. (Ed.). Mamíferos do Paraná. Pelotas: USEB, 2009. p. 201-238.

CHEREM, J. J. Registros de mamíferos não voadores em estudos de avaliação ambiental no sul do Brasil. Biotemas, Florianópolis, v. 18, n. 2, p. 169-202, 2005.

CHEREM, J. J.; ALTHOFF, S. L.; SIMÕES-LOPES, P. C.; GRAIPEL, M. E. Lista dos mamíferos do estado de Santa Catarina, sul do Brasil. Mastozoologia Neotropical, Mendoza, v. 11, n. 2, p. 151-184, 2004.

CHEREM, J. J.; GRAIPEL, M. E.; TORTATO, M.; ALTHOFF, S.; BRÜGGEMANN, F.; MATOS, J.; VOLTOLINI, J. C.; FREITAS, R.; ILLENSEER, R.; HOFFMANN, F.; GHIZONIJR, I. R.; BEVILACQUA, A.; REINICKE, R.; SALVADOR, C. H.; FILIPPINI, A.; FURNARI, N.; ABATI, K.; MORAES, M.; MOREIRA, T.; OLIVEIRA-SANTOS, L. G. R.; KUHNEN, V.; MACCARINI, T.; GOULART, F.; MOZERLE, H.; FANTACINI, F.; DIAS, D.; PENEDO-FERREIRA, R.; VIEIRA, B. P.; SIMÕESLOPES, P. C. Mastofauna terrestre do Parque Estadual da Serra do Tabuleiro, Estado de Santa Catarina, sul do Brasil. Biotemas, Florianópolis, v. 24, n. 3, p. 73-84, 2011.

CHRISTOFF, A. U.; PETERS, F. B.; ROTH, P. R. O.; COELHO, E. L.; JUNG, D. M. H. Lagomorpha e Myomorpha. In: WEBER, M. M.; ROMAN, C.; CÁCERES, N. C. (Ed.). Mamíferos do Rio Grande do Sul. Santa Maria: Editora UFSM, no prelo.

CIMARDI A. V. Mamíferos de Santa Catarina. Florianópolis: Fundação de Amparo à Tecnologia e Meio Ambiente, 1996. 302 p. CORTI, M.; MERANI, M. S.; VILLAFANE, G. Multivariate morphometrics of vesper mice (Calomys): preliminary assessment of species, population, and strain divergence. Zeitschrift für Säugetierkunde, Berlin, v. 52, n. 1, p. 236-242, 1987.

FERREIRA, F. Aspectos da vegetação e do uso do solo da microrregião costeira sul do Estado de Santa Catarina, Brasil. 2006. 140 f. Dissertação (Mestrado em Biologia Vegetal) Universidade Federal de Santa Catarina, Florianópolis. 2006.

FORMOSO, A. E.; SAUTHIER, D. E. U.; PARDIÑAS, U. F. J. Mammalia, Rodentia, Sigmodontinae, Holochilus brasiliensis (Desmarest, 1819): distribution extention. Check List, Viçosa, v. 6, n. 2, p. 195-197. 2010.

GARDNER, A. L.; PATTON, J. L. Karyotypic variation in oryzomyine rodents (Cricetinae) with comments on chromosomal evolution in the Neotropical cricetine complex. Occasional Papers of the Museum of Zoology, Ann Arbor, v. 49, n. 1, p. 1-48, 1976.

GONZÁleS, E. M.; LANFRANCO, J. A. M. Mamíferos de Uruguay - guía de campo e introducción a su estudio y conservación. Montevideo: Banda Oriental/Vida Silvestre/MNHN, 2010. 464 p.

GRAIPEL, M. E.; CHEREM, J. J.; XIMENEZ, A. Mamíferos silvestres não voadores da Ilha de Santa Catarina, sul do Brasil. Biotemas, Florianópolis, v. 14, n. 2, p. 109-140, 2001. 
HERSHKOVITZ, P. South American marsh rats, genus Holochilus, with a summary of sigmodont rodents. Fieldiana: Zoology, Chicago, v. 37, n. 1, p. 639-673, 1955.

HERSHKOVITZ, P. Evolution of neotropical Cricetine rodents (Muridae) with special reference to the Phyllotine group. Fieldiana: Zoology, Chicago, v. 46, n. 1, p. 1-524, 1962.

IBGE - INSTITUTO BRASILEIRO DE GEOGRAFIA E ESTATÍSTICA. Mapa de Vegetação do Brasil - Esc. 1:5.000.000. 2004. Disponível em: <ftp://ftp.ibge.gov.br/Cartas_e_Mapas/ Mapas_Murais/>.Acesso em: 20 jan. 2012.

KRAVETZ, F. O.; BUSCH, M.; PERCICH, R. E.; MANJÓN, M. C.; MARCONI, P. Ecología de Calomys laucha (Rodentia, Cricetidae) en el departamento de Río Cuarto (Córdoba). Criterios para determinación de dedas y crescimento. Ecología, Madri, v. 6, n. 1, p. 35-44, 1981.

MARQUES, R. V. O gênero Holochilus (Mammalla: Cricetidae) no Rio Grande do Sul: taxonomia e distribuição. Revista Brasileira de Zoologia, Curitiba, v. 4, n. 4, p. 347-360, 1988.

MASSOIA, E. Caracteres y rasgos bioecológicos de Holochilus brasiliensis chacarius Thomas ("rata nutria") de la provincia de Formosa y comparaciones con Holochilus brasiliensis vulpinus (Brants) (Mammalia - Rodentia - Cricetidae). Revista de Investigaciones Agropecuarias. Serie 1, Biología y Producción Animal, Buenos Aires, v. 8, n. 1, p. 13-40, 1971.

MASSOIA, E. El estado sistemático de cuatro especies de cricetidos sudamericanos y comentarios sobre otras especies congenericas (Mammalia-Rodentia). Ameghiniana, Buenos Aires, v. 17, n. 1, p. 280-287, 1980.

MASSOIA, E. El estado sistematico y zoogeografia de Mus brasiliensis Desmarest y Holochilus sciureus Wagner (MammaliaRodentia-Cricetidae). Physis, Buenos Aires, v. 39, n. 1, p. 31-34, 1981.

MASSOIA, E.; FORNES, A.; WAINBERG, R. L.; DE FRONZA, T. G. Nuevos aportes al conocimiento de las especies bonaerenses del género Calomys (Rodentia-Cricetidae). Revista de Investigaciones Agropecuarias. Serie 1, Biología y Producción Animal, Buenos Aires, v. 5, n. 4, p. 63-92, 1968.

MUSSER, G. G.; CARLETON, M. D. Superfamily Muroidea. In: WILSON, D. E; REEDER, D. M. (Ed.). Mammal species of the world: a taxonomic and geographic reference. 2. ed. Washington: Smithsonian Institution Press, 2005. p. 894-1522.

OLIVEIRA, J. V.; BONVICINO, C. R. Ordem Rodentia. In: REIS, N. R.; PERACCHI, A. L.; PEDRO, W. A.; LIMA, I. P. (Ed.). Mamíferos do Brasil. 2 ed. Londrina: Edifurb, 2011. p. 358-415.

PAGLIA, A. P.; FONSECA, G. A. B.; RYLANDS, A. B.; HERRMANN, G.; AGUIAR, L. M. S.; CHIARELlO, A. G.; LEITE, Y. L. R.; COSTA, L. P.; SICILIANO, S.; KIERULFF, M. C. M.; MENDES, S. L.; TAVARES, V. C.; MITTERMEIER, R. A.; PATTON, J. L. Lista anotada dos mamíferos do Brasil/ Annotated checklist of Brazilian mammals. Occasional Papers in Conservation Biology, Washington, n. 6, p. 1-76, 2012.

PASSAMANI, M.; CERBONCINI, R. A. S.; OLIVEIRA, J. E. Distribution extension of Phaenomys ferrugineus (Thomas, 1894), and new data on Abrawayaomys ruschii Cunha and Cruz, 1979 and Rhagomys rufescens (Thomas, 1886), three rare species of rodents (Rodentia: Cricetidae) in Minas Gerais, Brazil. Check List, Viçosa, v. 7, n. 6, p. $827-831,2011$.
PATTERSON, B. D. Patterns and trends in the discovery of new Neotropical mammals. Diversity and Distributions, Matieland, v. 6, n. 1, p. 145-151, 2000.

PEARSON, O. P.; PATTON, J. L. Relationships among South American Phyllotine rodents based on chromosome analysis. Journal of Mammalogy, Lawrence, v. 57, n. 1, p. 677-686, 1976. PERCEQUillo, A. R.; WEKSLER, M.; COSTA, L. P. A new genus and species of rodent from the Brazilian Atlantic Forest (Rodentia: Cricetidae: Sigmodontinae: Oryzomyini), with comments on Oryzomyine biogeography. Zoological Journal of the Linnean Society, London, v. 161, n. 1, p. 357-390, 2011.

PETERS F. B.; ROTH, P. R. O.; MACHADO, L. F.; COELHO, E. L.; JUNG, D. M. H.; CHRISTOFF, A. U. Assembléia de mamíferos dos agroecossistemas constituintes da bacia hidrográfica do rio da Várzea, Rio Grande do Sul. Biotemas, Florianópolis, v. 23, n. 4, p. 91-107, 2010.

ROCHA, R. G.; FERREIRA, E.; LEITE, Y. L. R.; FONSECA, C.; COSTA, L. P. Small mammals in the diet of Barn owls, Tyto alba (Aves: Strigiformes) along the mid-Araguaia River in central Brazil. Zoologia, Curitiba, v. 28, n. 6, p. 709-716, 2011.

SAUTHIER, W. O. U.; ABBA, A. M.; SAUTHIER, D. E. U. Nests of Oligoryzomys sp. and Holochilus brasiliensis (Rodentia, Cricetidae) in eastern Entre Ríos Province, Argentina. Mastozoología Neotropical, Mendoza, v. 17, n. 1, p. 207-211, 2010.

SCHEIBLER, D. R.; CHRISTOFF, A. U. Habitat associations of small mammals in southern Brazil and use of regurgitated pellets of birds of prey for inventorying a local fauna. Brazilian Journal of Biology, São Carlos, v. 67, n. 4, p. 619-625, 2007.

SILVA, F. Mamíferos silvestres do Rio Grande do Sul. Porto Alegre: Fundação Zoobotânica do Rio Grande do Sul, 1994. 245 p. TAYLOR, I. Barn owls: predator-prey relationships and conservation. Cambridge: Cambridge University Press, 1994. 311 p.

TESTONI, A. F.; FUMis, J.; ALThOFF, S. A.; TORTATO, F. R.; CHEREM, J. J. Akodon serrensis Thomas, 1902 (Mammalia: Rodentia: Sigmodontinae): Records in Santa Catarina State, southern Brazil. Check List, Viçosa, v. 8, n. 6, p. 1344-1346, 2012. VELARDE, E.; AVILA-FLORES, R.; MEDELLÍN, R. A. Endemic and introduced vertebrates in the diet of barn owl (Tyto alba) on two island of the Gulf of California, Mexico. The Southwestern Naturalist, Memphis, v. 60, n. 2, p. 284-290, 2007.

VIVO, M.; CARMIGNOTTO, A. P.; GREGORIN, R.; HINGSTZAHER, E.; IACK-XIMENES, G. E.; MIRETZKI, M.; PERCEQUILLO, A. R.; ROLLO, M. M.; ROSSI, R. V.; TADDEI, V. A. Checklist of mammals from São Paulo State, Brazil. Biota Neotropica, Campinas, v. 11, n. 1a, p. 1-21, 2010.

VOSS, R. S. An introduction to the Neotropical muroid rodent genus Zygodontomys. Bulletin of the American Museum of Natural History, New York, v. 210, n. 1, p. 1-113, 1991.

WEKSLER, M.; PERCEQUILO, A. R.; VOSS, R. S. Ten new genera of Oryzomyine rodents (Cricetidae: Sigmodontinae). American Museum Novitates, New York, n. 3537, p.1-29, 2006.

WILLIAMS, D. F.; MARES, M. A. A new genus and species of Phyllotine rodent (Mammalia: Muridae) from northwestern Argentina. Annals of Carnegie Museum, Pittsburg, v. 47, n. 1, p.193-221, 1978. 